\title{
Evaluation of the use of serum C-reactive protein concentration to predict outcome in puppies infected with canine parvovirus
}

\author{
Vanessa McClure, BVsc; Mirinda van Schoor, BVsc, MMedVet; Peter N. Thompson, Bvsc, MMedVet, PhD; \\ Mads Kjelgaard-Hansen, DVM, PhD; Amelia Goddard, BVsc, MMedVet
}

\begin{abstract}
Objective-To evaluate associations of serum C-reactive protein (CRP) concentration with duration of hospitalization and with outcome in puppies with canine parvoviral enteritis.

Design-Prospective observational study.

Animals-79 client-owned puppies with naturally acquired canine parvovirus infection.

Procedures-All puppies received supportive care. Serum CRP concentration was measured at the time of admission, approximately every 10 to 12 hours for the first 48 hours, and then every 24 hours until discharge from the hospital or death. Associations between outcome and CRP concentration at various time points or changes in CRP concentration over time were assessed via multiple logistic regression. Associations of CRP concentration with survival time and duration of hospitalization among survivors were estimated with Cox proportional hazards regression. Use of CRP concentration to predict outcome was evaluated by means of receiver operating characteristic curve analysis.
\end{abstract}

Results-Serum CRP concentrations at admission and 12 and 24 hours later were positively associated with odds of death, and CRP concentrations at 12 and 24 hours after admission were negatively associated with survival time for puppies. Among survivors, duration of hospitalization was positively associated with CRP concentrations at 12, 24, and 36 hours after admission. Sensitivity and specificity of CRP concentration to differentiate between survivors and nonsurvivors at 24 hours after admission were $86.7 \%$ and $78.7 \%$, respectively (considered moderately accurate).

Conclusions and Clinical Relevance-Although serum CRP concentration was associated with outcome in puppies with canine parvovirus enteritis, it did not prove to be a good predictor of outcome when used alone. (J Am Vet Med Assoc 2013;243:361-366)

C anine parvovirus first emerged in the mid-1970s as an enteric pathogen of $\operatorname{dogs}^{1-3}$ and has remained a major pathogen worldwide. The characteristic signs of CPV enteritis include hemorrhagic diarrhea, vomiting, loss of appetite, lethargy, fever, severe dehydration, sudden collapse, and death. ${ }^{4-6}$ It has been reported that the chances of death are higher in CPV-infected puppies that have evidence of systemic inflammation at the time of admission, compared with those that do not. ${ }^{7}$ The systemic inflammatory response is thought to be attributable to bacterial trans-

From the Departments of Companion Animal Clinical Studies (McClure, van Schoor, Goddard) and Production Animal Studies (Thompson), Faculty of Veterinary Science, University of Pretoria, Onderstepoort 0110, South Africa; and the Department of Veterinary Clinical and Animal Sciences, Faculty of Health and Medicine, University of Copenhagen, 1870 Frederiksberg, Denmark (Kjelgaard-Hansen). Dr. McClure's present address is Onderstepoort Veterinary Academic Hospital, Department of Companion Animal Clinical Studies, Faculty of Veterinary Science, Onderstepoort 0110, South Africa.

This manuscript represents a portion of a thesis submitted by Vanessa McClure to the University of Pretoria Faculty of Veterinary Science as partial fulfillment of the requirements for a Master of Veterinary Medicine degree.

Supported by the Faculty of Veterinary Science, University of Pretoria, Onderstepoort, South Africa.

Presented in abstract form at the Annual Congress of the European College of Veterinary Internal Medicine, Tolouse, France, September 2010 The authors thank Mandy Albertyn and Sarina Myburgh for help with sample collection and Cheryl Booth for laboratory assistance.

Address correspondence to Dr. McClure (vanessa.mcclure@up.ac.za).

\begin{tabular}{ll}
\hline & \multicolumn{1}{c}{ AbBREVIATIONS } \\
APP & Acute-phase protein \\
AUC & Area under the curve \\
CPV & Canine parvovirus \\
CRP & C-reactive protein \\
ROC & Receiver operating characteristic \\
TEM & Transmission electron microscopy \\
\hline
\end{tabular}

location from the damaged intestinal tract and resulting coliform septicemia. ${ }^{8}$ Escherichia coli was recovered from the lungs or liver of $90 \%$ of puppies that had deaths attributed to parvovirus infection, and pathological findings compatible with acute respiratory distress syndrome were also reported, ${ }^{9}$ providing evidence that bacterial translocation and coliform septicemia are important factors in CPV infection. Endotoxin is a potent stimulator of inflammatory responses through activation of a cytokine-mediated procoagulant effect on endothelial cells..$^{10,11}$

To date, no agent-specific treatment has proven effective for CPV enteritis. The survival fraction of infected puppies has been reported to be as low as $9 \%$ if the condition is left untreated. ${ }^{7}$ Treatment and convalescent periods may be prolonged and consequently expensive, with mortality rates of $4 \%$ to $48 \%{ }^{12}$; therefore, many clients may elect for euthanasia of their pets. Thus, an accurate predictor of morbidity and death for puppies with CPV infection 
would be of benefit. Several studies have been performed to investigate potential prognostic markers in dogs infected with CPV, which include endocrine,$^{13}$ hematologic, ${ }^{14}$ and biochemical variables. ${ }^{7,15}$ In human patients with severe sepsis, mortality rates may exceed $30 \%$, despite advanced supportive care ${ }^{16}$; these results are similar to those of studies in which mortality rates of $15 \%{ }^{17}$ to $53 \%{ }^{15}$ were found for parvovirus-infected puppies that received aggressive supportive care. Associations between serum concentrations of CRP, a major APP in humans and dogs, and survival have been investigated in humans with severe sepsis. ${ }^{16}$ Median serum CRP concentrations were significantly higher in nonsurvivors, compared with the survivors, indicating that CRP concentration could be used as a predictor of outcome in human patients with sepsis. ${ }^{16}$

In dogs, CRP is primarily produced in the liver by de novo synthesis and released into the bloodstream following stimulation by proinflammatory cytokines. ${ }^{16,18}$ The extent of changes in circulating CRP concentrations during the course of disease seems to reflect underlying disease progression and inflammatory activity. ${ }^{1,20, a}$ Circulating CRP concentrations have been shown to increase in dogs with various diseases such as pancreatitis, neoplasia, sepsis, ehrlichiosis, leptospirosis, and leishmaniasis. ${ }^{21-26}$ Results of 1 study ${ }^{15}$ indicated that mean serum CRP concentration in parvovirus-infected puppies at the time of hospital admission was higher in nonsurvivors than in survivors. Interpretation of changes in serum CRP concentration over time, together with other hematologic and biochemical variables, has been found to be useful in assessing the severity of various diseases in dogs. ${ }^{27}$ The primary objective of the study reported here was to evaluate the association of serum CRP concentrations over time with morbidity and death in puppies with canine parvoviral enteritis. Our primary interest was to determine whether serial serum CRP concentrations in puppies could be used to predict outcome (survival or death). A secondary objective was to determine the usefulness of serum CRP concentrations to predict the duration of hospitalization for these dogs.

\section{Materials and Methods}

Animals-Client-owned puppies that were naturally infected with CPV and admitted to the isolation ward of the Onderstepoort Veterinary Academic Hospital, Faculty of Veterinary Science, University of Pretoria, South Africa, were recruited for a prospective, observational study between November 27, 2008, and September 25,2009 . Puppies of any breed and either sex that had severe clinical signs associated with CPV infection (eg, dehydration, anorexia, lethargy, vomiting, and hemorrhagic diarrhea) necessitating hospitalization and aggressive treatment were eligible for enrollment. The diagnosis of CPV infection was confirmed through TEM of feces, ELISA for canine parvoviral antigen, or both. Other enteric viruses (eg, coronavirus or canine distemper virus) were also excluded by TEM. Puppies with ob- vious signs of other inflammatory processes (eg, obvious wounds or respiratory or skin diseases) were excluded. A thin peripheral blood smear from each puppy was examined at the time of admission to screen for overt hematologic evidence of parasitemia (eg, Babesia spp or Ehrlichia spp). A fecal sample was also evaluated by examination of a wet mount and fecal flotation for the presence of Giardia spp, Isospora spp, and helminth ova on admission. If any of these parasites were found, those puppies were excluded from the study. Written consent was received from the owners prior to enrollment of puppies in the study. The research protocol was approved by the University of Pretoria Animal Use and Care Committee.

All puppies were treated according to a standard supportive care plan for dogs with parvovirus infection, which was adapted from a previously described treatment regimen. ${ }^{14}$ All dogs received IV crystalloid fluids, antiemetics,

Table 1-Comparison of age, sex, and body weight for 79 puppies of various breeds with naturally acquired CPV enteritis that did ( $n=63$ ) or did not (16) survive to discharge.

\begin{tabular}{|c|c|c|c|}
\hline Variable & Survivors & Nonsurvivors & $P$ value* \\
\hline $\begin{array}{l}\text { Age (mo) } \\
\text { Weight (kg) }\end{array}$ & $\begin{array}{c}4(2-6) \\
5.8(4.2-10.4)\end{array}$ & $\begin{array}{c}5(3-6) \\
8.3(4.4-12.3)\end{array}$ & $\begin{array}{l}0.352 \\
0.267\end{array}$ \\
\hline $\begin{array}{l}\text { Sex } \\
\text { Male } \\
\text { Feme }\end{array}$ & 37 & $\begin{array}{c}11 \\
5\end{array}$ & $\begin{array}{c}0.572 \\
-\end{array}$ \\
\hline $\begin{array}{l}\text { Data are } \\
{ }^{*} \text { Age an } \\
\text { rank sum tes } \\
\text { exact test. } \\
\quad=\text { Not }\end{array}$ & $\begin{array}{l}\text { an (interquarti } \\
\text { ht differences } \\
\text { differences in } \\
\text { able. }\end{array}$ & $\begin{array}{l}\text { nge) or number } \\
\text { re evaluated wi } \\
\text { were evaluatec }\end{array}$ & $\begin{array}{l}\text { gs. } \\
\text { Wilcoxon } \\
\text { the Fisher }\end{array}$ \\
\hline
\end{tabular}

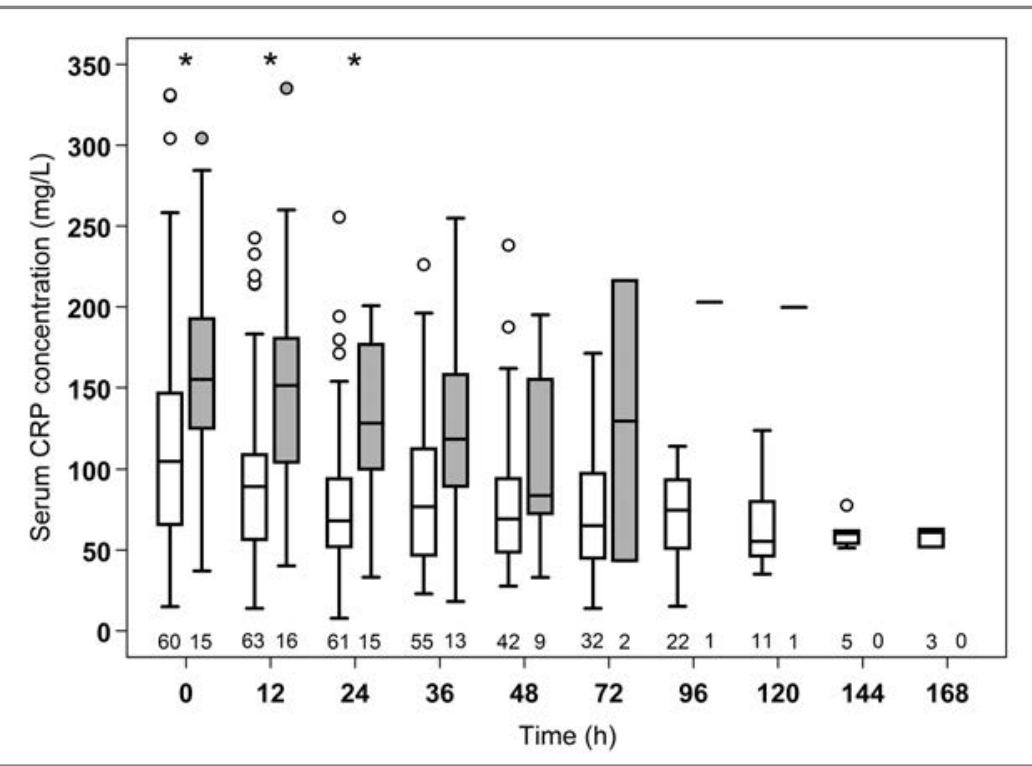

Figure 1-Box-and-whiskers plot of $\log _{2}$-transformed serum CRP concentrations in 79 puppies with CPV enteritis that did (white boxes) or did not (gray boxes) survive to discharge. Serum CRP concentration was measured at the time of admission, approximately every 10 to 12 hours for the first 48 hours, and then at 24-hour intervals. The box incorporates the middle $50 \%$ of observations; horizontal lines within each box represent the median value. The whiskers extend to the smallest and largest observations that are $<1.5$ times removed from the interquartile range. Outliers are plotted separately as circles. Time 0 was the time of admission, before any treatments were administered; numbers above the x-axis indicate number of samples from each group (samples at the time of admission were missing from 3 dogs that survived and 1 that died). ${ }^{*}$ Mean CRP concentration was significantly $(P<0.05)$ different between survivors and nonsurvivors. 
and antimicrobials. Analgesics were given as needed. Any changes in treatment were administered at the discretion of the attending clinician. Puppies were discharged when they had stopped vomiting completely (no vomiting for 2 days), Hct and serum total protein concentration were within or close to the respective reference ranges, and puppies were consuming their daily caloric requirements independently. Clinicians were blinded to the results of CRP measurement.

Procedures-A clinical examination was performed on each puppy at admission and then once daily until discharge from the hospital or death. The fecal sample collected at admission was refrigerated immediately and submitted $\leq 12$ hours after collection for examination with TEM. Fecal samples from most puppies were also tested with a point-of-care ELISA ${ }^{\mathrm{b}}$ for CPV antigen according to the manufacturer's instructions.

At admission (prior to any treatment) and twice daily (approx 10 to 12 hours apart) thereafter for the first 48 hours, blood (approx 3 to $5 \mathrm{~mL}$ ) was collected via jugular venipuncture into a serum separator tube. ${ }^{c}$ After 48 hours, blood samples were collected every 24 hours until discharge or death. Serum samples were left to clot at room temperature (approx $25^{\circ} \mathrm{C}$ ) and then centrifuged at 2,100 X $g$ for 10 minutes. A minimum of $100 \mu \mathrm{L}$ of serum was required for CRP analysis. Serum was collected and stored at $-80^{\circ} \mathrm{C}$ until it could be analyzed as a batch, to avoid interassay variability. Samples were stored for up to 10 months (CRP concentrations reportedly remain stable in serum stored at $-20^{\circ} \mathrm{C}$ for up to a year in a controlled laboratory setting and up to 34 months in a research setting ${ }^{28}$ ). The CRP concentrations were measured with an automated turbidimetric immunoassay for human $\mathrm{CRP}^{\mathrm{d}, \mathrm{e}}$ previously validated for use in dogs. ${ }^{29,30}$ The assay was calibrated with commercially available purified canine $\mathrm{CRP}^{\mathrm{f}}$ to ensure species-specific measurement of CRP with the heterologous assay. ${ }^{29}$ The intra-assay coefficient of variation for the CRP method, by means of immunoturbidimetry, was calculated as 0.1 . The detection range for the assay was 5.1 to $163.3 \mathrm{mg} / \mathrm{L}$. Samples that exceeded the detection limit during initial testing were manually diluted to a $1: 3$ or $1: 5$ ratio in saline $(0.9 \%$ $\mathrm{NaCl}$ ) solution at the discretion of the laboratory technician. Internal controls consisting of pooled serum samples from 5 healthy dogs were routinely run with the batch of samples. Samples were analyzed as a batch, and all outliers were immediately reanalyzed to confirm results.

Statistical analysis-Data were analyzed with commercially available statistical software. ${ }^{g}$ Serum CRP concentrations were log-transformed to achieve normal distribution and are reported as geometric means. At each time point, mean concentrations were compared between survivors (dogs that lived to be discharged from the hospital) and nonsurvivors by use of a Student $t$ test. Associations between $\log _{2}$ CRP concentration or change over time in $\log _{2}$ CRP concentration and outcome (survival vs death) were determined by multiple logistic regression, adjusted for age, body weight, and sex. Change over time was expressed as the ratio of CRP concentrations at 2 time points. Separate models were used to evaluate CRP concentrations at the time of admission and 12, 24, and 36 hours after admission and to detect changes in CRP concentration from admission to 12 and 24 hours after admission. Associations between $\log _{2}$ CRP concen- trations at the described time points and survival time were estimated by means of Cox proportional hazards regression, adjusted for age, weight, and sex. For dogs that survived, the association between $\log _{2}$ CRP concentrations and duration of hospitalization was estimated in a similar manner. Fit of the logistic regression models was assessed with a Hosmer-Lemeshow goodness-of-fit test, and the proportional hazards assumption of the Cox regression models was assessed with Schoenfeld residuals. The clinical value of CRP concentration to predict the outcome of death was evaluated by means of ROC curves. Death of a dog attributed to CPV infection was classified as a truepositive result, whereas survival to discharge was classified as a true-negative result. The AUC was calculated and compared among time points; sensitivity, specificity, Youden index $(\mathrm{J}=$ sensitivity + specificity -1$)$, and likelihood ratios were calculated for all possible CRP concentration cutoff values. Values of $P<0.05$ were considered significant for all statistical tests.

Table 2-Summary of results for multiple logistic regression analysis of associations between $\log _{\text {-transformed serum CRP }}$ concentration and death in 79 hospitalized puppies with CPV enteritis.

\begin{tabular}{lccc}
\hline Predictor & OR & $\mathbf{9 5 \%} \mathbf{C l}$ & P value \\
\hline Serum CRP concentration & & & \\
Admission & 2.34 & $1.04-5.27$ & 0.041 \\
$12 \mathrm{~h}$ & 4.28 & $1.57-11.7$ & 0.005 \\
$24 \mathrm{~h}$ & 4.93 & $1.71-14.2$ & 0.003 \\
$36 \mathrm{~h}$ & 1.89 & $0.86-4.17$ & 0.115 \\
Change in serum CRP & & & \\
$\quad$ concentration & & & \\
$\quad$ vs 12 h & 1.78 & $0.56-5.64$ & 0.327 \\
$\quad$ vs 24 h & 1.20 & $0.59-2.43$ & 0.619 \\
12 vs 24 h & 1.00 & $0.32-3.10$ & 0.993 \\
\hline
\end{tabular}

Serum CRP concentration was determined with an automated turbidimetric immunoassay in samples collected at the time of admission (prior to any treatment), twice daily thereafter (approx 10 to 12 hours apart) for 48 hours, and then every 24 hours until discharge or death; times of sample collection shown in the table are approximate. Concentrations were log-transformed to achieve normal distribution. The ORs estimate the effect of a higher (2-fold difference) CRP concentration on the odds of death after adjustment for age, body weight, and sex.

Table 3-Summary of results of Cox proportional hazards regression analysis for associations of $\log _{2}$-transformed serum CRP concentration with survival time and duration of hospitalization in 79 puppies with CPV enteritis.

\begin{tabular}{|lccc|}
\hline Outcome and predictor & Hazard ratio & $\mathbf{9 5 \%} \mathbf{C l}$ & $\boldsymbol{P}$ value \\
\hline $\begin{array}{l}\text { Survival time (event = death) } \\
\text { Serum CRP concentration }\end{array}$ & & & \\
$\quad$ Admission & 1.82 & $0.91-3.64$ & 0.089 \\
$12 \mathrm{~h}$ & 3.61 & $1.59-8.20$ & 0.002 \\
$24 \mathrm{~h}$ & 4.90 & $1.88-12.8$ & 0.001 \\
$36 \mathrm{~h}$ & 1.61 & $0.76-3.44$ & 0.217 \\
Hospitalization time & & & \\
(event = discharge) & & & \\
$\quad$ Serum CRP concentration & 0.84 & $0.65-1.09$ & 0.198 \\
$\quad$ Admission & 0.63 & $0.44-0.90$ & 0.012 \\
$12 \mathrm{~h}$ & 0.53 & $0.36-0.78$ & 0.001 \\
$24 \mathrm{~h}$ & 0.53 & $0.36-0.79$ & 0.002 \\
$\quad 36 \mathrm{~h}$ & & \\
\hline Hazard ratios estimate the effect of a higher (2-fold difference) \\
serum CRP concentration on instantaneous hazard of the event \\
(death or discharge) after adjustment for age, body weight, and sex. \\
See Table 2 for remainder of key. \\
\hline
\end{tabular}




\section{Results}

Seventy-nine puppies (31 sexually intact females and 48 sexually intact males) of various breeds were enrolled in the study. Ages ranged from 1 to 18 months. Age, body weight, and sex distribution were not significantly different between survivors and nonsurvivors (Table 1). Sixteen of 79 (20\%) puppies died; median survival time for nonsurvivors was 3 days after admission (range, 1 to 6 days).

Serum CRP concentrations at admission; approximately 12, 24, 36, and 48 hours later; and then every 24 hours up to 168 hours were summarized and compared between puppies grouped according to outcome (survivors and nonsurvivors; Figure 1). Samples collected at admission were missing from 3 dogs that survived and 1 that died. Geometric mean CRP concentrations for survivors at the time of admission and 12 and 24 hours after admission were 100.6, 81.3 , and $67.6 \mathrm{mg} / \mathrm{L}$, respectively, and for nonsurvivors, concentrations were 146.3 , 140.1, and $116.1 \mathrm{mg} / \mathrm{L}$, respectively. Values were significantly different between groups for these 3 time points. Results of the multiple logistic regression analysis were summarized (Table 2). A higher CRP concentration (2-fold difference) at admission $(P=0.041), 12$ hours after admission $(P=0.005)$, or 24 hours after admission $(P=0.003)$ was associated with greater odds of death. However, there was no significant association between the odds of death and changes in CRP concentration between admission and 12 hours $(P=0.327)$, admission and 24 hours $(P=0.619)$, or 12 and 24 hours $(P=0.993)$.

Results of Cox proportional hazards regression analysis were summarized (Table 3). Survival time was negatively associated with serum CRP concentration at $12(P=0.002)$ and 24 hours $(P=$ 0.001 ), indicating that higher CRP concentrations were significantly associated with shorter survival times. Among dogs that survived, duration of hospitalization was positively associated with CRP concentration at $12(P=0.012), 24(P=$ $0.001)$, and 36 hours $(P=0.002)$ after admission, indicating that higher CRP concentrations were associated with a longer hospitalization time.

Evaluation of ROC curves (Figure 2) for the ability to predict outcome (survival vs death) on the basis of serum CRP concentrations revealed no significant differences among AUCs for CRP concentration at admission and 12, 24, and 36 hours later; however, the model in which CRP concentration had the greatest apparent ability to discriminate between survivors and nonsurvivors was at 24 hours after admission (AUC, $0.79 ; 95 \%$ confidence interval, 0.68 to 0.87 ). Cutoff values for the various time points were evaluated (Figure 3). When CRP concentration at 24 hours after admission was used as a predictor of death, the Youden index was maximized at a cutoff of $97.3 \mathrm{mg} / \mathrm{L}$, with a sensitivity and specificity of $86.7 \%$ and $78.7 \%$, respectively, and a positive likelihood ratio of 4.1 with positive and negative predictive values of $50 \%$ and $96 \%$, respectively (at the 20\% mortality rate in this study). Greater specificity could be achieved with higher cutoff values to reduce false-positive results: at cutoff values $>140 \mathrm{mg} / \mathrm{L}$,

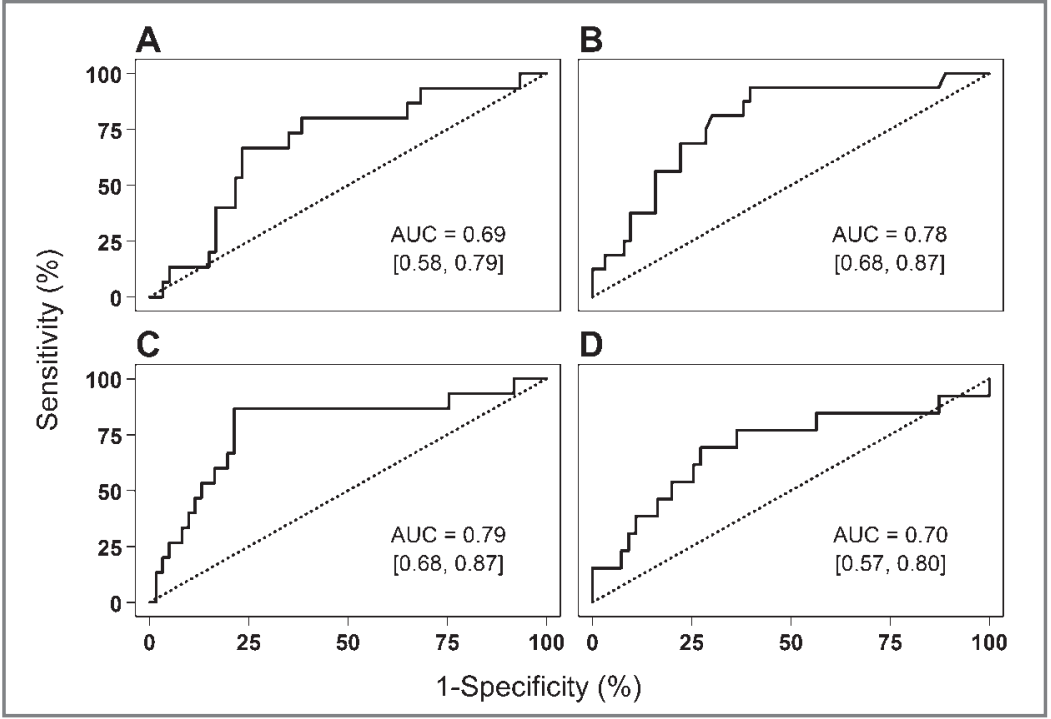

Figure 2-Receiver operating characteristic curves for the ability to predict outcome (ie, discrimination between puppies with CPV infection that did or did not survive to discharge) on the basis of $\log _{2}$-transformed serum CRP concentration at the time of admission (A) and at 12 (B), 24 (C), and 36 (D) hours after admission. The dotted $45^{\circ}$ line represents the line of no discrimination. Numbers in square brackets represent $95 \%$ confidence limits for the AUC.

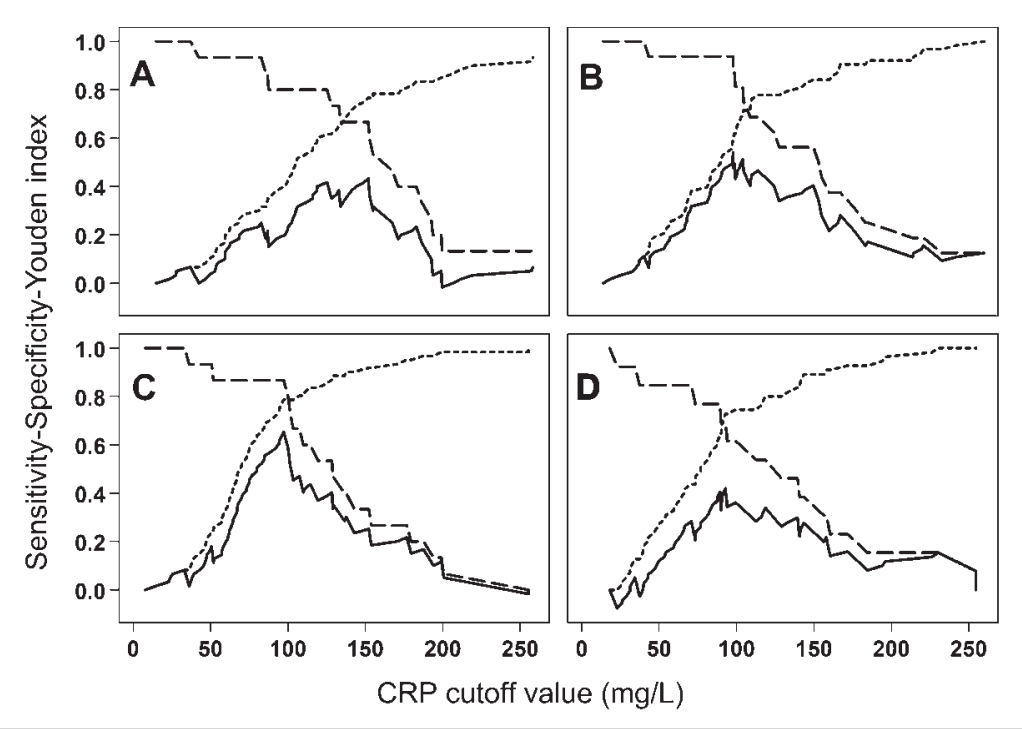

Figure 3-Plots of sensitivity (dashed line), specificity (dotted line), and Youden index (solid line) for use of $\log _{2}$-transformed CRP concentration at admission (A) and 12 (B), 24 (C), and 36 (D) hours after admission to predict outcome in puppies with CPV infection. A true positive was defined as a dog that died and for which death was attributed to CPV infection; a true negative was defined as a dog that survived to discharge. Youden index = sensitivity + specificity -1 . 
specificity was $>90 \%$. However, the low sensitivity at these cutoffs indicated very few true-positive results; therefore, the positive predictive value remained approximately $50 \%$ and the positive likelihood ratio also did not increase. At 12 and 24 hours after admission, the negative predictive value (ie, prediction of survival) remained $>95 \%$ for cutoff values up to approximately $100 \mathrm{mg} / \mathrm{L}$.

\section{Discussion}

In the study reported here, serum CRP concentrations were evaluated in puppies with CPV enteritis at the time of hospital admission, twice daily thereafter (approx 10 to 12 hours apart) for 48 hours, and then every 24 hours until discharge or death. Results indicated that higher serum CRP concentrations (2-fold difference) at 12 and 24 hours after admission were associated with shorter survival times in these patients, compared with those that had lower CRP concentrations. The results also revealed that survivors with higher CRP concentrations at 12, 24 and 36 hours after admission had longer hospitalization periods than did those with lower CRP concentrations. A CRP concentration cutoff value of $97.3 \mathrm{mg} / \mathrm{L}$ at 24 hours after admission had a sensitivity of $86.7 \%$ and a specificity of $78.7 \%$ to predict death on the basis of results of ROC curve analysis and Youden index calculations. The AUC at 24 hours was 0.79 , which is regarded as a moderately accurate diagnostic test; a highly accurate test should have an AUC $>0.90 .^{31}$ A significant association was not found when investigating changes in CRP concentrations over time between survivors and nonsurvivors.

The finding of a significant positive association between serum CRP concentrations (at admission and 12 and 24 hours later) and the odds of death in puppies with CPV enteritis was not surprising; the half-life of CRP is constant under all conditions so that the sole determinant of serum concentration is the synthesis rate, which directly reflects the intensity of the pathological process that causes production of CRP. ${ }^{32}$ The rates of lymphoid and intestinal cell turnover appear to be the main factors that indicate the severity of CPV infection. High turnover rates reflect high rates of virus replication and cell destruction. ${ }^{6}$ Cell destruction results in the release of inflammatory cytokines that stimulate production of CRP in the liver. When the stimulus is removed, the circulating CRP concentration is rapidly reduced. ${ }^{32}$ The most notable feature of CRP is the rapidity of its appearance and increased concentration in serum following an acute stimulus. ${ }^{19}$ The increase in CRP concentration in dogs is more rapid than that in humans. Increased concentrations are first detected 4 hours after a stimulus and increase to peak values at approximately 24 hours in dogs, whereas CRP concentrations in humans are first detected after 6 hours, with peak concentrations occurring at approximately 48 hours. ${ }^{21,32}$ Because plasma CRP concentrations change rapidly, frequent blood collection was indicated for the present study. The lack of a significant difference between survivors and nonsurvivors when changes in CRP over time were evaluated was unexpected because the half-life of CRP is short; however, a similar result was found in a study ${ }^{33}$ performed to evaluate CRP concentrations in dogs infected with $\mathrm{Ba}$ besia spp. In dogs with CPV enteritis, this finding may be attributable to severe tissue damage and systemic inflammation present, which may persist for an extended period.
The prognostic value of serum APPs was evaluated in dogs with CPV infection at the time of hospital admission in another study. ${ }^{15}$ Serum CRP, ceruloplasmin, and haptoglobin concentrations were reported to be higher in dogs with CPV infection than in control dogs, but the magnitude of increase in CRP concentration from baseline values was higher than that for the other APPs. The CRP concentrations at admission were higher in puppies that died, compared with those that survived. ${ }^{15}$ These findings were in agreement with the results of our study. Investigators of the previous study ${ }^{15}$ also reported that a circulating CRP concentration of $92.4 \mathrm{mg} / \mathrm{L}$ at the time of admission had a sensitivity and specificity of $91 \%$ and $61 \%$, respectively, to differentiate survivors from nonsurvivors. Those results differ from the results of our study, where a cutoff value of 131.3 $\mathrm{mg} / \mathrm{L}$ was determined at admission, with a sensitivity and specificity of $73.3 \%$ and $65 \%$, respectively. Our study differed in that CRP concentration was measured serially, and a cutoff of $97.3 \mathrm{mg} / \mathrm{L}$ at 24 hours after admission appeared to have greater sensitivity and specificity $(86.7 \%$ and $78.7 \%$, respectively) than did values at admission. This may be because puppies were first evaluated at different times in the course of their illness (some seen soon after the onset of clinical signs, whereas others may have been ill for a day or more), but after 24 hours of treatment, their physiologic status was more standardized.

Various studies have been performed to evaluate other variables as predictors of outcome in CPVinfected puppies. Results of 1 study ${ }^{14}$ indicated that an accurate prognosis could be obtained for puppies with CPV enteritis at 24 hours after admission by evaluating changes in blood leukocyte concentrations. Investigators in another study ${ }^{7}$ found that CPV-infected puppies that have lymphopenia and hypoalbuminemia at admission are hospitalized for longer periods, compared with puppies without these abnormalities. Evaluation of endocrine hormones in puppies with CPV enteritis, including serum cortisol and thyroxine concentrations, found that high serum cortisol and low thyroxine concentrations at 24 and 48 hours after admission were associated with death. ${ }^{13}$ The use of serum CRP concentrations for prognostic purposes may have some advantages over these other variables. Administration of some drugs as well as variations in breed and body size have been reported to affect circulating concentrations of thyroid hormones and cortisol in healthy dogs. ${ }^{34-36}$ However, CRP concentrations are not known to be affected by administration of drugs, ${ }^{32}$ and CRP concentrations do not differ significantly among dogs of different breeds and body sizes. ${ }^{37}$ In response to inflammation, CRP concentration increases rapidly. Compared with changes in WBC counts, which do not always reflect the severity of disease, CRP concentration may be a more stable variable for monitoring throughout the disease process because it is not subjected to bone marrow responses or fluctuations resulting from extravasation. ${ }^{26}$ Despite the significant association between serum CRP concentrations and odds of death in the present study, results of ROC curve analysis indicated that the discriminative ability of CRP concentration alone in predicting outcome was only moderately accurate. This is in accordance with findings of several oth- 
er clinical studies ${ }^{27,38-40}$ that indicated circulating CRP concentration alone does not correspond to outcome.

A limitation of the present study was that the investigators had no control over when puppies were brought to the clinic during the course of the disease process. This could have affected the CRP results at admission as well as patient outcome. Modifications of the standard supportive treatment by different attending clinicians may have had an effect on the results, but this is unlikely because the treatment is not agent specific and does not affect the pathogenesis of the disease.

Prognostication remains a challenging topic. During the past 20 years, much research has been done to find ways of improving the precision and accuracy of clinicians' estimates. ${ }^{16}$ Results of the study reported here indicate that the use of serum CRP concentration alone to make decisions about outcome is not reliable, but serial measurements of serum CRP may prove useful to monitor the progression of the disease and adapt treatment accordingly. Future studies are warranted to evaluate serial CRP concentrations in conjunction with other variables proven to be of value in prognostication for dogs with $\mathrm{CPV}$ infection, such as leukocyte count and results of serum biochemical and endocrine hormone analysis.

a. Kjelgaard-Hansen M, Strom H, Mikkelsen LF, et al. Grading of surgical trauma by means of canine $\mathrm{C}$-reactive protein measurements (abstr). Vet Clin Pathol 2008;37:6.

b. Antigen Rapid CPV Ag test kit, Animal Genetics Inc, GyeonggiDo, Korea.

c. Vacuette, Greiner Bio-One GmbH, Kremsmünster, Austria.

d. Alpha Wassermann Inc, Woerden, The Netherlands

e. Ace Alera Orb Diagnostics, Johannesburg, South Africa.

f. Life Diagnostics Inc, West Chester, Pa.

g. Stata, version 11.1, StataCorp, College Station, Tex.

\section{References}

1. Carmichael LE, Binn LN. New enteric viruses in the dog. Adv Vet Sci Comp Med 1981;25:1-37.

2. Carmichael LE. An annotated historical account of canine parvovirus. J Vet Med B Infect Dis Vet Public Health 2005;52:303-311.

3. Apple MJG, Cooper BJ, Greisen H, et al. Status report: canine viral enteritis. J Am Vet Med Assoc 1978;173:1516-1518.

4. Pollock RVH, Coyne MJ. Canine parvovirus. Vet Clin North Am Small Anim Pract 1993;23:555-568.

5. Smith-Carr S, Macintire DK, Swango LJ. Canine parvovirus. Part I. Pathogenesis and vaccination. Compend Contin Educ Pract Vet 1997;19:125-133.

6. Goddard A, Leisewitz AL. Canine parvovirus. Vet Clin North Am Small Anim Pract 2010;40:1041-1053.

7. Kalli I, Leontides LS, Mylonakis ME, et al. Factors affecting the occurrence, duration of hospitalization and final outcome in canine parvovirus infection. Res Vet Sci 2010;89:174-178.

8. Otto CM, Drobatz KJ, Soter C. Endotoxemia and tumor necrosis factor activity in dogs with naturally occurring parvovirus enteritis. J Vet Intern Med 1997;11:65-70.

9. Turk J, Miller M, Brown T, et al. Coliform septicemia and pulmonary disease associated with canine parvoviral enteritis: 88 cases (1987-1988). J Am Vet Med Assoc 1990;196:771-773.

10. Levi M, Van Der Poll T, Ten Cate H, et al. The cytokine-mediated imbalance between coagulant and anticoagulant mechanisms in sepsis and endotoxaemia. Euro J Clin Invest 1997;27:3-9.

11. Weiss DJ, Rashid J. The sepsis-coagulant axis: a review. J Vet Intern Med 1998;12:317-324.

12. Prittie J. Canine parvoviral enteritis: a review of diagnosis, management, and prevention. J Vet Emerg Crit Care 2004;14:167-176

13. Schoeman JP, Goddard A, Herrtage ME. Serum cortisol and thyroxine concentrations as predictors of death in critically ill puppies with parvoviral diarrhea. J Am Vet Med Assoc 2007;231:1534-1539.

14. Goddard A, Leisewitz AL, Christopher MM, et al. Prognostic usefulness of blood leukocyte changes in canine parvoviral enteritis. J Vet Intern Med 2008;22:309-316.

15. Kocaturk M, Martinez S, Eralp O, et al. Prognostic value of serum acute-phase proteins in dogs with parvoviral enteritis. J Small Anim Pract 2010;51:478-483.

16. Memi D, Gursoy O, Tasdogan M, et al. High C-reactive protein and low cholesterol levels are prognostic markers of survival in severe sepsis. J Clin Anesth 2007;19:186-191.

17. Macintire DK, Smith-Carr S. Canine parvovirus. Part II. Clinical signs, diagnosis, and treatment. Compend Contin Educ Pract Vet 1997;19:291-302.

18. Kushner I, Feldmann G. Control of the acute phase response. Demonstration of C-reactive protein sysnthesis and secretion by hepatocytes during acute inflammation in the rabbit. J Exp Med 1978;148:466-477.

19. Caspi D, Baltz ML, Snel F, et al. Isolation and characterization of C-reactive protein from the dog. Immunology 1984;53:307-313.

20. Kjelgaard-Hansen M, Strom H, Mikkelsen LF, et al. Canine serum Creactive protein as a quantitative marker of the inflammatory stimulus of aseptic elective soft tissue surgery. Vet Clin Pathol 2013;in press.

21. Conner JG, Eckersall PD, Ferguson J, et al. Acute phase response in the dog following surgical trauma. Res Vet Sci 1988;45:107-110.

22. Rikihisa Y, Yamamoto S, Kwak I, et al. C-reactive protein and alpha 1-acid glycoprotein levels in dogs infected with Ehrlichia canis. J Clin Microbiol 1994;32:912-917.

23. Shimada T, Ishida Y, Shimizu M, et al. Monitoring C-reactive protein in Beagle dogs experimentally inoculated with Ehrlichia canis. Vet Res Commun 2002;26:171-177.

24. Jergens AE, Schreiner CA, Frank DE, et al. A scoring index for disease activity in canine inflammatory bowel disease. J Vet Intern Med 2003;17:291-297.

25. Holm JL, Rozanski EA, Freeman LM, et al. C-reactive protein concentrations in canine acute pancreatitis. J Vet Emerg Crit Care 2004; 14:183-186.

26. Ceron JJ, Eckersall PD, Martynez-Subiela S. Acute phase proteins in dogs and cats: current knowledge and future perspectives. Vet Clin Pathol 2005;34:85-99.

27. Gebhardt C, Hirschberger J, Rau S, et al. Use of C-reactive protein to predict outcome in dogs with systemic inflammatory response syndrome or sepsis. J Vet Emerg Crit Care (San Antonio) 2009;19:450-458.

28. Brindle E, Fujita M, Shofer J, et al. Serum, plasma and dried blood spot high sensitivity C-reactive protein enzyme immunoassay for popular research. J Immunol Methods 2010;362:112-120.

29. Kjelgaard-Hansen M. Comments on measurement of C-reactive protein in dogs. Vet Clin Pathol 2010;39:402-403.

30. Kjelgaard-Hansen M, Jensen AL, Kristensen AT. Evaluation of a commercially available human C-reactive protein (CRP) turbidometric immunoassay for determination of canine serum CRP concentration. Vet Clin Pathol 2003;32:81-87.

31. Greiner M, Pfeiffer D, Smith RD. Principles and practical application of the receiver-operating characteristic analysis for diagnostic tests. Prev Vet Med 2000;45:23-41.

32. Pepys MB, Hirschfield GM. C-reactive protein: a critical update. J Clin Invest 2003;111:1805-1812.

33. Köster LS, Van Schoor M, Goddard A, et al. C-reactive protein in canine babesiosis caused by Babesia rossi and its association with outcome. J S Afr Vet Assoc 2009;80:87-91.

34. Regnier A, Toutain PL, Alvinerie M, et al. Adrenocortical function and plasma biochemical values in dogs after subconjunctival treatment with methylprednisolone acetate. Res Vet Sci 1982;32:306-310.

35. Daminet $S$, Ferguson DC. Influence of drugs on thyroid function in dogs. J Vet Intern Med 2003;17:463-472.

36. Reimers TJ, Lawler DF, Sutaria PM, et al. Effects of age, sex, and body size on serum concentrations of thyroid and adrenocortical hormones in dogs Am J Vet Res 1990;51:454-457.

37. Couto CG, Ceron JJ, Parra MD et al. Acute phase protein concentrations in retired racing Greyhounds. Vet Clin Pathol 2009;38:219-223.

38. Mitchell KD, Kruth SA, Wood RD, et al. Serum acute phase protein concentrations in dogs with autoimmune hemolytic anemia. J Vet Intern Med 2009;23:585-591.

39. Griebsch C, Arndt G, Raila J, et al. C-reactive protein concentration in dogs with primary immune-mediated hemolytic anemia. Vet Clin Pathol 2009;38:421-425.

40. Glare P, Sinclair C, Downing M, et al. Predicting survival in patients with advanced disease. Eur J Cancer 2008;44:1146-1156. 